\title{
Anorexia Nervosa as Irreducibly Normative: A Dilemma Posed for Dominic Murphy's Two- Stage Picture of Psychiatry
}

\author{
Juliette Croce $^{1}$
}

\begin{abstract}
In Psychiatry in the Scientific Image, philosopher Dominic Murphy proposes a two-stage picture of psychiatry with mental disorders conceptualized as an underlying malfunction of a cognitive mechanism, followed by a judgement of the harmful effects this malfunction can have. Murphy himself raises a dilemma to this mechanistic reductionism, in that there are several mental disorders which seem to be irreducibly normative, and that the disorders must make normative assumptions of rationality in order to be fully understood. In this paper, I take up this obstacle to the two-stage picture by arguing that the three key aspects of anorexia nervosa - its triggers, aims, and the sustaining behaviors - render it irreducibly normative under Murphy's model. I contend that the first two components of triggers and aims cannot be reduced down to a psychological malfunction of rationality in light of evidence that their causal phenomenology is largely culturally-based and deemed rationally oriented towards human flourishing. I then analyze potential underlying biological and physiological factors of the disorder's self-starvation behaviors to assess whether this is enough to reduce anorexia down to a mechanistic explanation. I conclude that normativity needs to be assessed for a complete conception of the disorder, such that it cannot be reduced down to a non-normative malfunctioning mechanism of rationality.
\end{abstract}

Keywords: Anorexia Nervosa, Instrumental Rationality, Content Rationality, Two-stage Model.

\footnotetext{
${ }^{1}$ Department of Psychology, McGill University. Address all correspondence to Juliette.croce@mail.mcgill.ca.
} 


\section{Introduction}

There has been much contention as to whether anorexia nervosa $(\mathrm{AN})$ is a culture-bound syndrome because of its relative prevalence in Western culture. It is this culture-boundedness that has motivated us to critically analyze the normative aspects of the disorder and whether they are integral to understanding it. More specifically, because AN is set within a broader culture that idealizes skinniness and dieting, we are particularly interested in whether this renders the disorder irreducibly normative. That is, what makes AN intriguing as a mental disorder is that it seems to operate according to a culturally normative threshold. For instance, certain behaviors are considered culturally normal and rational, such as following diets or possessing a desire to meet a beauty standard of thinness. However, this can become something considered disordered, despite the same culture encouraging the key components of this supposed deviance - the ideal of thinness and diet culture itself.

This goes against the two-stage picture of psychiatry that Dominic Murphy proposes, in which mental disorders should be conceptualized as an underlying malfunction of a cognitive mechanism, followed by a judgment of the harmful effects said malfunction can have. His proposal attempts to reduce mental disorders to objective scientific facts within the first stage, with the evaluation of the disorders bracketed as a separate matter in the second stage (Murphy 2006, 151). In disorders caused by lesions, for example, it is easy to define a causal relationship between the lesioned cognitive structure and the disorder that follows from this malfunctioning structure. However, for most mental disorders, mechanistic explanations like this are insufficient. Murphy himself raises this as a potential dilemma against his two-stage picture with regards to disorders that necessarily hinge on behavior deemed 'irrational,' as these disorders seem to be irreducibly normative. In other words, they cannot be reduced to mechanistic cognitive malfunctions because their malfunction irrational behavior - is formed from subjective ideas of what one ought to do or how one ought to do things. Murphy demonstrates this dilemma in an analysis of addiction as a disorder that must make 
normative assumptions of rationality to be fully understood (Murphy 2006). In this paper, we present a similar analysis of this dilemma as it presents itself in the disorder of AN.

We aim to examine how this prescription of skinniness as the ideal standard of beauty - a standard that is potentially culture-bound - is necessary to understand the causes and sustainment of AN. Moreover, we seek to determine how both content-based rationality something deemed rational in itself - and instrumental rationality - something deemed rational because it is what needs to be done to achieve some goal - fits within the disorder, and how this problematizes the two-stage picture Murphy puts forth. We argue that although there are potential physiological factors that influence the development of AN, cultural-level factors and ideals of what is deemed as constituting a 'good life' contradict solely mechanistic explanations of the illness. A 'good life' in this sense includes the association of thinness with success, popularity, or general contentment. More specifically, ideals of thinness and attributes associated with this beauty standard are intrinsic to an adequate conception of the disorder. We contend that while the triggers and goals of AN are less contentiously irreducibly normative, the behaviors of self-starvation can potentially be removed from this normativity. However, at this point, there is insufficient evidence to support that $\mathrm{AN}^{\prime} \mathrm{s}$ behaviors can be reduced down to malfunctions in mechanisms of rationality, and hence the disorder remains irreducibly normative.

We will first describe the origins, characteristics, and perpetuating factors of the disorder. We will also present a brief background on the picture of rationality that Murphy presents, fleshing out key differences in instrumental and content rationality for analyzing irreducibly normative disorders. From there, we will lay out the three components found within the modern conception of $\mathrm{AN}$ : that of the triggers of the disorder, the goals of the disorder, and the behaviors that sustain the disorder. We will analyze how each component functions within ideas of instrumental rationality and content rationality, and consider the issues they present within a mechanistic explanation 
of AN. For the first two components triggers and goals - we argue that they cannot be reduced down to a psychological malfunction of rationality because their causal phenomenology is largely culturally-based and, importantly, deemed rationally oriented towards human flourishing. Additionally, in looking at a review of potential underlying biological and physiological factors of self-starvation - including genetic factors, personality predispositions, and perception-based issues such as body dysmorphia - we will assess whether this is enough to reduce anorexic disorders to a mechanistic malfunction. We conclude that evidence of underlying factors is insufficient and that normativity is an integral piece required for the conceptualization of AN.

\section{Background}

$\mathrm{AN}$ is an eating disorder with three main clinical features. First, the disorder is marked by significantly low weight considering factors such as age, height and development, weight history, medical complications, and the speed of the weight loss (Call, Attia and Wash 2017, 2072). Second, the individual shows an intense fear of gaining weight accompanied by a relentless pursuit of thinness. Consequently, the individual engages in behaviors aimed at weight loss or preventing weight gain, including food intake restriction, purging, and compulsive exercise (Call, Attia and Wash 2017, 2065). The disorder is also characterized by body dysmorphia or disturbances in one's body shape or weight experience, and typically a perception of one's own body as too large. Individuals with AN often fail to recognize the severity of the illness and the health risk it poses, which is compounded by the way the striving for thinness takes over the individual's selfschema. For example, if the self-schema of an individual with AN involves a persistent belief that they are not thin enough, the individual may be more attuned to cues that confirm this belief such as social media trends showing weight loss 'progress' or diets that encourage low calorie intake to look a certain way - and disregard cues that contradict it - such as concerns from others or health issues arising from the disorder. 
$\mathrm{AN}$ is further categorized into two types: restrictive or binge eating and purging. Those with restrictive AN exhibit behaviors of limiting daily food intake via counting calories and portion sizes, avoiding specific food items or classes of food, eating only at certain times or after a certain amount of time has passed since the last meal, adopting rules that govern eating behavior, and intensive exercise. The binge eating and purging type of anorexia is characterized by a binge of large amounts of food, followed by a purge induced by postbinge guilt. This can take the form of vomiting, use of laxatives or diuretics, or excessive exercise (Call, Attia and Walsh 2017, 2072). Though presenting similar behaviors, this subtype differs from bulimia nervosa due to the abnormally low weight criteria required for an anorexia diagnosis. However, both bulimia nervosa and anorexia nervosa are themselves separated from other eating disorders such as binge eating disorder in that they feature a relentless drive for thinness.

Our goal in choosing $\mathrm{AN}$ as a psychiatric case study is due to the way that the defining characteristics of AN are often considered problematic within some cultures. Ironically, these same cultures emphasize and encourage dieting and idealize thinness. I will comprehensively assess the specific problems this poses for understanding $\mathrm{AN}$ as a malfunction of rationality, but it is important to briefly note what this culturally-specific dilemma entails. Culture-bound syndromes are those that are rooted in cultural values, involving the core norms of a specific culture (Banks, $1992,868)$. If a disorder is culture-bound, it is said to be more prevalent in a particular culture. Thus, if the motivation for self-starvation in individuals with AN is correlated to Western or North American culture in this case, it contradicts the idea that $\mathrm{AN}$ can be reduced to a rational malfunction. Importantly, this does not imply causation. For example, restricting eating to certain times is a common behavior in individuals with restrictive AN, but this is also a key element of the popular and accepted 'non-diet' of intermittent fasting found commonly within Western culture. That is, the behavior is pathologized in the former but not in the latter case. The question is thus if the similar activity 
within AN is irreducibly related to this accepted and seemingly rational behavior. Alternatively, it needs to be shown that this behavior can somehow be considered a different, irrational behavior problematic only in the cases of AN. In other words, if the disorder is correlated to cultural norms, then it cannot be reduced to a mechanistic malfunction because there is an important external component to it. It is important to note that we are arguing that a mere correlation, rather than causation, is enough to eliminate the possibility that AN can be understood without considering these normative factors in its description. To analyze this further, we will look at each of the elements of AN to see whether they can be characterized as instrumentally irrational and/or irrational in content.

First, we must understand instrumental and content rationality referred to above. We aim to relocate the ways that disorders involving apparent malfunctions of rationality can problematize mechanistic reductionism, as pointed to by Murphy in his example of addiction. While some argue that disorders based around malfunctioning conceptions of rationality and rational orientations can be reduced to a nonnormative, procedural domain of facts, Murphy points out that this is only seemingly possible if rationality is limited to instrumental rationality. Instrumental rationality is conceptualized as the rational behavior of pursuing certain actions for something to be realized, or an end goal to be attained. It is silent about the end goals and their characteristics themselves, and instead assesses whether the means used to realize these goals are themselves rational (Murphy 2006, 155). That is, a person with a goal is instrumentally rational in this regard if they act to achieve their goal. For example, a person can be said to be rational if they arrive at the bus stop a few minutes early before their bus is supposed to arrive because they are acting in a way to achieve the goals of avoiding missing the bus. This follows the normative format of 'if you don't want to miss the bus, then you ought to be at the bus stop a few minutes before it arrives.' Someone who does not act in a way to achieve these goals, thereby not following the principles laid out within this normative domain of rationality, is thus said to be irrational. 
Crucially, if this logic applies to understanding mental disorders, then it would be possible to get rid of the normativity described above because instrumental rationality is not substantively normative. Considering the example above, the normative aspect of what one 'ought' to do can be bracketed and replaced with the general idea that a person who doesn't want to miss the bus will arrive a few minutes early. The instrumental rationality held by people, then, is reduced to a domain of wellfunctioning psychological capacities because it can be translated into mechanistic claims of rationality. Everything a rational person does is not what one ought to do to achieve a goal, but is instead is what simply has to be done to achieve a goal. That is, if someone is acting outside of this procedural domain of rationality, then there is a failure in their mechanisms of said rationality. In the case of $\mathrm{AN}$, to be characterized as a mechanistic malfunction of instrumental rationality, people with AN would need to display a failure to achieve an end skinniness - by certain means - selfstarvation. This is difficult to claim because their starvation itself is a well- known means to the end of extreme thinness, and thus can be said to be instrumentally rational. This will be discussed more in-depth in the following sections.

Moreover, this description relies on the characterization of mental disorders' deviations from rationality as instrumental, as these are the deviations that can be translated into a domain of non-value laden descriptions. Murphy argues that this can be problematic because it ignores a broader understanding of rationality and irrationality featured in many psychological disorders, including AN. Most pertinently, it fails to address the issues of content rationality. Some disorders seem irrational because the fundamental desires that guide them are themselves perceived by the observer to be irrational. Murphy contends that desires deemed fundamentally rational are assessed according to what makes life good or flourishing $(2006,156)$. That is, content rationality is focused on whether goals are irrational in themselves, subject to reasoned scrutiny guided by ideals of 'human flourishing.' However, content rationality, unlike instrumental 
rationality, is irreducibly normative because of this relationship to what is described as 'human flourishing.' If a disorder is said to be irrational because the beliefs that guide it are themselves considered irrational - based on ideas of what constitutes human flourishing then that necessarily means normative assumptions are being made. For instance, Murphy shows that addiction presents this dilemma because what is targeted as the irrationality of addiction is its incompatibility with human flourishing, "...since the addict is in thrall to the lowest part of his nature" (Murphy 2006, 191). Therefore, addiction becomes subject to moral scrutiny rather than being reductively mechanistic.

We contend that AN presents a similar dilemma, specifically in the triggers and goals of the disorder being irreducibly tied to contentions of what constitutes human flourishing - namely, the idealization of weight loss and a beauty standard of thinness. It is only in the third factor of sustaining selfstarvation that there is the potential for instrumental and content irrationality to be guiding it. However, this alone does not mean that anorexia nervosa can be reduced to a mechanistic explanation because the potential mechanistic malfunctions undergirding the behaviors of self-starvation have yet to be adequately researched. Thus, in exploring how instrumental and content rationality function within each component of $\mathrm{AN}$, we conclude that a solely mechanistic malfunctioning undergirding the disorder cannot be deduced.

\section{(Ir)rationality of the Components of}

\section{Anorexia Nervosa}

To showcase how AN problematizes the two-stage picture by introducing irreducible normativity in its characterization, we will focus on the three key components to the disorder in this section. First are the triggers of the disorder, or the precipitating events that can potentially cause anorexia nervosa to take form in an individual. We argue that this component meets neither the criteria of being irrational in the instrumental sense, nor in the content-based sense because of the ideals of thinness associated with the 'good life.' The second element we examine is the goals of the disorder, i.e., the aims of thinness and weight gain avoidance that characterize 
AN. Similar to the first component, we argue that this element cannot fall into the category of being instrumentally or content-wise irrational because of the culture-boundedness of the aims. Lastly, we will discuss the behaviors of anorexia nervosa, those actively sustaining selfstarvation as the means to achieve the end goal of thinness. We argue that this is the component which crosses the threshold into the realm of what could be considered irrational. However, after considering causal explanations such as physiology or predisposing factors, we contend that there is not enough evidence to confirm that this irrationality can be reduced to a mechanistic explanation. Thus, we argue that $\mathrm{AN}$ remains irreducibly normative.

\section{Triggers of the Disorder}

The events that precipitate the development of anorexia nervosa are said to be one of the key components to the disorder (Call, Attica, and Walsh 2017, 2071). These events are conceptualized as triggers that provoke the key behaviors of a disorder (Abraham 2016, 50). In AN, the most common triggers are events that lead to an initial weight loss, including participation in sports that place an emphasis on low weight, deciding to go on a diet, or the stress of puberty (Call, Attia, and Walsh 2017, 2071). These triggers are key to a causal analysis of the disorder, as it is hypothesized that a potential cause of this disorder is the way in which precipitating events trigger predisposing traits of vulnerability to take hold, resulting in the illness (Call, Attica, and Walsh 2017, 2070).

Essential to understanding the role triggers play in $\mathrm{AN}$ and the question of whether they are rational in kind is that they do not necessarily require intent to reach the state of self-starvation that characterizes the disorder. Moreover, they do not need to be based on an aim for initial weight loss itself (Abraham 2016, 104). For example, a trigger may be a viral illness that was situational but caused the individual to lose significant weight meaning the weight loss was not sought out. Similarly, an individual begins practicing a sport because of interest in the sport, not an interest in the weight loss associated with the sport. If there is an intention for weight loss, the triggers often take the form of a decision to eat healthier and improve fitness, rather than 
lose an extreme amount of weight (105). However, the reason why the triggers play a crucial role in the initial weight loss developing into anorexic behaviors is that they allow for, in theory, both internal and external positive reinforcement to occur. Internally, self-confidence in one's body may arise, enhancing a preoccupation with the association between weight loss and body image (104). This is supported by external reinforcement in the form of compliments regarding the initial weight loss, thereby encouraging continued weight loss efforts

It could be argued that those that fall victim to these triggers are instrumentally rational by adopting the following rationale: 'If you want to achieve skinniness, you ought to lose weight.' That is, the individual is acting in a rational manner by losing weight to increase skinniness because that is what was internally and externally reinforced. The content rationality of these triggers that cause the initial weight loss is also present, such as the rational desire to eat healthily or play a sport. Moreover, because a desire doesn't even need to be present as in the example of the viral illness that can cause weight loss - rationality may not even be involved with this component of AN. Thus, AN can be understood as a rational and socially acceptable desire for thinness.

\section{Goals of the Disorder}

In addition to the triggering mechanism that causes the initial weight loss, AN is characterized by certain goals. These goals revolve around achieving and maintaining a certain weight, relentlessly striving for thinness, and avoiding weight gain (Call, Attia, and Walsh 2017, 2065). It is important to note that this aim of thinness coincides with other individual goals, often underlying and less noticeable. These proxy-goals include psychological explanations for desiring thinness, such as using weight loss as a mechanism of control to escape from feelings of anxiety (Abraham 2016, 43). They also include social explanations that point to the cultural tie between slimness and success, attractiveness, health, or popularity (Abraham 2016, 42). However, these remain a proxy to the main aim of obtaining low weight, which is the primary motivation of the transition from the initial trigger weight loss to anorexic behavior. 
To examine whether the goals of AN are rational or not, we must consider the constant and persistent concern over one's weight, and the role this plays in the individual's self-schema. If the fear of weight gain and pursuit of slimness is acceptable or normalized within a society, then this disorder cannot be reduced to an irrationality. This does seem to be the case, as studies find a relationship between culture and weight concern as a motivating factor (Keel and Klump 2003, 755). For example, this was shown in a study that examined the influence of Western media on the prevalence of eating disorders. In this study, Western media was introduced into the nation of Fiji, previously with little access to such media, and an increased prevalence of eating disorders was found (Call, Attia, and Walsh, 2071). In this way, the environmental influences mirrored industrialized Western cultures that emphasize the importance of thinness, with research showing that the majority of women in North American society share a preoccupation with body weight and dieting (Banks 1992, 872). This is not to say that a culture that emphasizes dieting and weight loss causes or necessitates the onset of anorexia nervosa - as noted, triggers are also a part of a causal explanation of the disorder - but that they play a role in the overall goal formation that guides self-starvation behaviors.

Similar to the triggers of the disorder, the fundamental goal of slimness does not meet the criteria to be considered irrational, instrumentally, or content-wise. If the motivating factor of weight concern is culture-bound, it may be said to be bound because of the way the aspiration for thinness is tied to the ' good life' - as pointed to in the noted association with popularity or success. The goal of AN is thus explained rationally in the following way: 'if you want to achieve said 'good life' then you ought to be skinny.' This 'good life' is framed in diet, self-help, and/or self-care trends that promise self-fulfillment, such as the intermittent fasting trend alluded to above. Therefore, the goals of the disorder can be said to be instrumentally rational because it offers an appropriate solution of skinniness to achieve what is idealized as the 'good life.' It is also rational content-wise, in that the fundamental desire to be skinny and avoid weight gain 
is a standard desired in Western societies. Therefore, because skinniness is the central ideal of beauty in Western culture, the fundamental preference for such skinniness cannot be reduced to a malfunction of rational preferences.

\section{Behaviors of the Disorder}

If $\mathrm{AN}$ is considered a malfunction of the mechanism of rationality, then this malfunction cannot be within the triggers, nor the aims of the disorder. As described above, these two components can be considered rational instrumentally and content-wise. However, there does seem to be something that doesn't fit with viewing AN as rational, because of the extreme amounts of harm it can do to an individual. Therefore, if there is a mechanistic failure in rationality, then it must originate from the behaviors that pathologize it and separate it from mere dieting or weight-focused behaviors considered as rational. In this section, we will look more closely at the sustainment of self-starvation as the key component of AN that potentially crosses from the rationality described above into a realm of irrationality. We will then look at whether this can be explained mechanistically by analyzing various potential causal explanations for such behaviors, including predispositions and physiological factors. We conclude that there isn't enough evidence to reduce the disorder to a disrupted rational mechanism.

The behaviors of AN include a compulsive need for maintaining a low weight or continual weight loss by selfstarvation tactics. As discussed earlier, this includes restricting food intake, purging, or compulsive exercise that decreases weight past the point of a healthy weight. It is this extreme food refusal behavior that distinguishes people with AN from those losing weight for health purposes, as individuals with AN may not be able to see or care about the unhealthy stage they reach (Banks 1992, 872). Similarly, the weight of individuals with AN may get so low it no longer 'fits' the idealized body type within cultural standards, and therefore the tactics to achieve such an ideal slimness can no longer be considered a rationalized action. That is, while it may be considered rational to follow a diet because that is what is prescribed to be healthy and meets the societal ideals of beauty and 
slimness, individuals with AN struggle to balance the health and beauty standards of human flourishing. Thus, their actions cannot be characterized as rational in achieving the 'good life.'

The irrationality of anorexia nervosa, therefore, seems to lie in the selfstarvation behaviors of restricting, purging, and compulsively exercising to achieve weight loss. Understood through instrumental rationality, these behaviors are formatted as follows: 'If you want to lose weight and achieve thinness, you ought to severely restrict food intake and/or exercise strenuously.' This is arguably irrational, as weight loss doesn't require such extreme measures to be taken. The fundamental desire that guides this - the content rationality of the anorexic behaviors - is also arguably irrational. That is, the goal to engage in self-starvation behaviors to continuously lose weight and avoid fatness past the point of healthiness, or the ideal standard of beauty seems irrational, in and of itself. The crucial question is whether this irrationality can be explained mechanistically, or whether the behavior can be reduced to a mechanistic malfunction of rationality.
There are a variety of potential causal explanations that connect irrationality to a predisposing or physiological factor. There have been several studies that suggest a genetic vulnerability in the development of the illness, demonstrated by an increased prevalence in twins and within family members (Call, Attia, and Walsh 2017, 2070). This suggests that there may be a combination of genetic and epigenetic factors - changes in behaviors or environments that affect gene expression - that increase the likelihood of developing the disorder following triggers discussed previously (Abraham 2016, 46). It has also been suggested that certain personality types are at an increased risk of developing the disorder, including avoidant, obsessivecompulsive, or anxious personalities (44).

However, if predisposing factors are the key causal connection between the malfunctioning of a mechanism of rationality and the development of $\mathrm{AN}$, two dilemmas remain. First, when considering predispositions alone, they fail to explain why the weight concern remains culture-bound. That is, if the disorder was reduced to these 
predispositions, then these weightoriented goals that guide AN should be more widespread across the globe. Second, the reduction of the disorder to a predisposing personality type removes the fact that these personality traits would, in other cases, not be seen as harmful. For example, even though people on the more extreme end of the spectrum of obsessive personalities have a higher likelihood to sustain self-starvation behaviors, this same trait isn't in itself disordered. Therefore, obsession with starvation and weight loss specifically needs to be considered as potentially problematic, not just the predisposition towards obsession itself. Precipitating events and key motivations, thus, need to be considered to conceptualize the disorder in its entirety.

Another causal explanation for sustaining self-starvation behaviors is the physiology of the individual. For instance, once an individual has restricted themselves for so long that they do not respond to internal messages to eat more to feel better, there may be an increase in opioid activity that leads to an elevation of mood (45). This, in turn, causes the individual to continue restricting because it makes them feel 'good.' This occurs similarly with strenuous exercise leading to a greater opioid release. In contrast, the starvation state also may lead to a low mood and increased obsession that maintains the illness (Call, Attia, and Walsh 2017, 2070). In either case, this points to a dysfunction in a neural mechanism of rationality that forms an association either between starvation and contentment, or between starvation and a consistently low mood neutralizing the negative feelings of being hungry. However, this fails to explain how an individual can reach this stage of the disorder in the first place. That is, in these physiological explanations, something remains external to these malfunctioning mechanisms, namely, the motivations which are potentially culture-bound and not intrinsic to a malfunction in rationality.

\section{Conclusion}

In this paper, we have shown that there has yet to be enough research establishing a clear underlying mechanistic malfunction in the behaviors of self-starvation that provides a complete causal explanation for AN. The science 
reviewed is therefore insufficient to reduce the behaviors of $A N$ to neural dysfunction. Moreover, AN cannot be characterized merely by the behaviors of self-starvation and the potential mechanistic malfunction that underlies said behaviors, because this disregards the triggers and primary motivations. That is, even though it is only the behavioral element of $\mathrm{AN}$ that has the potential to split from normativity - with both the triggers and motivations behind the disorder failing to be held up as necessarily irrational because of their cultural normativity - the disorder cannot be conceptualized by these behaviors on their own.

For this reason, it has been suggested that the disorder requires a combined explanation involving precipitating events, potential predispositions, and perpetuating factors (Abraham 2016, 50). To understand the full etiology of the disorder, it needs to be noted that the triggers and aims cannot be reduced down to malfunctions in a neural mechanism of rationality. Rather, there is a certain irreducible normativity in the conceptualization of the disorder including the potential culture- boundedness that inherently ties the disorder to societal standards of the 'good life' as well as external triggers relying upon norms of dieting and accepted weight-loss behaviors. This means that, despite the potential mechanistic explanations of the irrationality of selfstarvation, the disorder cannot be reconstructed in a way to get rid of this normativity completely, and is incapable of being isolated to a set of facts and neural mechanisms.

This presents a problem for the two-stage picture developed by Murphy. Since the disorder cannot be reduced down to a neural dysfunction in rationality, the disorder fails to fit within the dysfunction component of Murphy's model. Rather, the disorder takes the form of a violation of content rationality - the irrational fundamental desire of selfstarvation - and instrumental rationality the self-starvation tactics which extend past healthy goals or a culturally-ideal body type - but this irrationality in behaviors fails to be reduced down to a mechanistic issue. This follows Murphy's claims about the problematics of disorders involving rationality, in that the mechanization of said rationality is 
doubted as possible. Thus, similar to addiction, AN can be said to be irrational in that it is incompatible with human flourishing, thereby becoming irreducible normative as part of moral scrutiny and with this normativity consistently haunting the diagnoses, as Murphy (2006) himself points to (197).

In conclusion, at this current point in scientific research, there is not a clear enough causal reduction to a mechanistic malfunction of rationality to explain the behaviors of AN without this explanation. In light of the other two irreducibly normative components of the disorder, this creates a dilemma in understanding $\mathrm{AN}$ - and mental illnesses more generally - as harmful dysfunctions of neural components presented in the two-stage picture. Importantly, this leads to an essential conceptualization of the disorder as a potentially normal functioning mind that sustains selfstarvation behaviors because of external cues, societal standards, or cultural malfunctioning. Whether AN can be considered a disorder and how it can be classified as such under the two-stage model is a problem yet to be resolved.

\section{$\underline{\text { References }}$}

Abraham, Suzanne. Eating Disorders: the Facts. Oxford: Oxford University Press, 2016. Accessed April 20, 2021. https://ebookcentral.proquest.com/lib/mcgill/detail.action?docID $=4083318$

Banks, Caroline G. "'Culture' in culture-bound syndromes: The case of anorexia nervosa." Social Science $\mathcal{E}$ Medicine 34, no. 8 (April 1992): 867-884. https://doi.org/10.1016/0277-9536(92)90256-P

Call, Christine C., Evelyn Attia, and B. Timothy Walsh. "Feeding and Eating Disorders." In Kaplan \& Sadock's Comprehensive Textbook of Psychiatry $10^{\text {th }}$ Edition, edited by Benjamin J. Sadock, Virginia A. Sadock, and Pedro Ruiz, 2065-2082. Philadelphia: Wolters Kluwer, 2017.

Keel, Pamela K. and Kelly L. Klump. “Are eating disorders culture-bound syndromes? Implications for conceptualizing their etiology." Psychological Bulletin 129, no. 5 (September 2003): 747-769. doi: 10.1037/0033-2909.129.5.747. PMID: 12956542. Madsen, Sarah K., Cara Bohon, and Jamie D. Feusner. "Visual Processing in anorexia nervosa and body dysmorphic disorder: similarities, differences, and future research directions." J Psychiatr Res 47, no. 10 (October 2013): 1483-1491. doi:10.1016/j.jpsychires.2013.06.003. PMID: 23810196. 
Murphy, Dominic. 2006. Psychiatry in the Scientific Image. Cambridge, Massachusetts: MIT Press, 2006. 